\title{
NEW INFRARED MEASUREMENTS OF MAGNETIC FIELDS ON COOL STARS
}

\author{
STEVEN H. SAAR \\ Center for Astrophysics, 60 Garden Street, Cambridge, MA 02138, U.S.A.
}

\begin{abstract}
I present a preliminary analysis of IR spectra of five $\mathrm{K}$ and $\mathrm{M}$ dwarfs and two RS CVn variables. Evidence for significant magnetic flux is found on several stars, a number of which are detected for the first time. Field strengths $(B)$ on the RS CVn variables are lower than in the active dwarfs, consistent with the concept of pressure balance limiting $B$ in stellar photospheres. I compare the results with previous measurements.
\end{abstract}

Key words: infrared: stars - stars: activity - stars: late-type - stars: magnetic fields

\section{Introduction and Observations}

Observations of stellar magnetic fields in the IR have three main advantages over the visible: (1) The ratio of the Zeeman splitting to the Doppler width, $\Delta \lambda_{B} / \Delta \lambda_{D} \propto \lambda$, increasing the ease of detection (e.g., Giampapa et al. 1983); (2) the line density is lower, so that blends are less likely to confuse a measurement; and (3) for cooler stars, the near IR is close to the stellar flux maximum. Advantages (2) and (3) are important for M dwarfs, and (1) is particularly helpful for low-gravity RS CVn systems, where attempts at optical field measurements have mostly failed (Marcy and Bruning 1984; see, however, Bopp et al. 1989). I have been obtaining IR spectra with the NOAO 4-m Fourier Transform Spectrometer (4-m FTS; Hall et al. 1979) since 1985. I present some recent results of this program, including new measurements for several active $\mathrm{K}$ and $\mathrm{M}$ dwarfs and RS CVn variables.

Observations with the 4-m FTS were made in May 1991 (except for EV Lac, made in Decmber 1985) using the $\mathrm{C}+\mathrm{D}$ detectors and an interference filter passing $4,400-4,600 \mathrm{~cm}^{-1}$ at an unapodized resolution of $\lambda / \Delta \lambda=64,000\left(0.07 \mathrm{~cm}^{-1}\right)$. Of primary interest in this wavelength region is a $\mathrm{Ti}$ I multiplet with $g_{\text {eff }}$ ranging from 1.125 to 2.5 . These lines first appear in early $\mathrm{K}$ stars, grow strong by late $\mathrm{K}$, and remain relatively strong at least to $\mathrm{M} 5$.

The spectra were reduced following Saar and Linsky (1985), with the exception that they were not apodized. While this leaves the spectra with a peculiar appearance due to the sinc function instrumental profile, it facilitates a more accurate extraction of telluric lines, and retains the full instrumental resolution. Spectra of inactive dwarfs and giants with similar spectral types (e.g., GL 273, M4 V; $\mu$ Gem, M3 III) were used to help in distinguishing weak blends. For display purposes, all spectra and models have been smoothed with a five-point running mean to suppress sinc ringing.

\section{Analysis and Discussion}

Visual inspection of the Ti I lines in active $\mathrm{M}$ dwarfs (Fig. 1) reveals profiles that are much too broad for the (optically) measured $v$ sin $i$. Compare, for example, AU Mic (Fig. 1, left: $8 \mathrm{~km} \mathrm{~s}^{-1}$; Vogt, Soderblom, and Penrod 1983) with HR 7275 (Fig. 2, right: $v \sin i=15 \mathrm{~km} \mathrm{~s}^{-1}$; Strassmeier et al. 1988). The lines are also too 


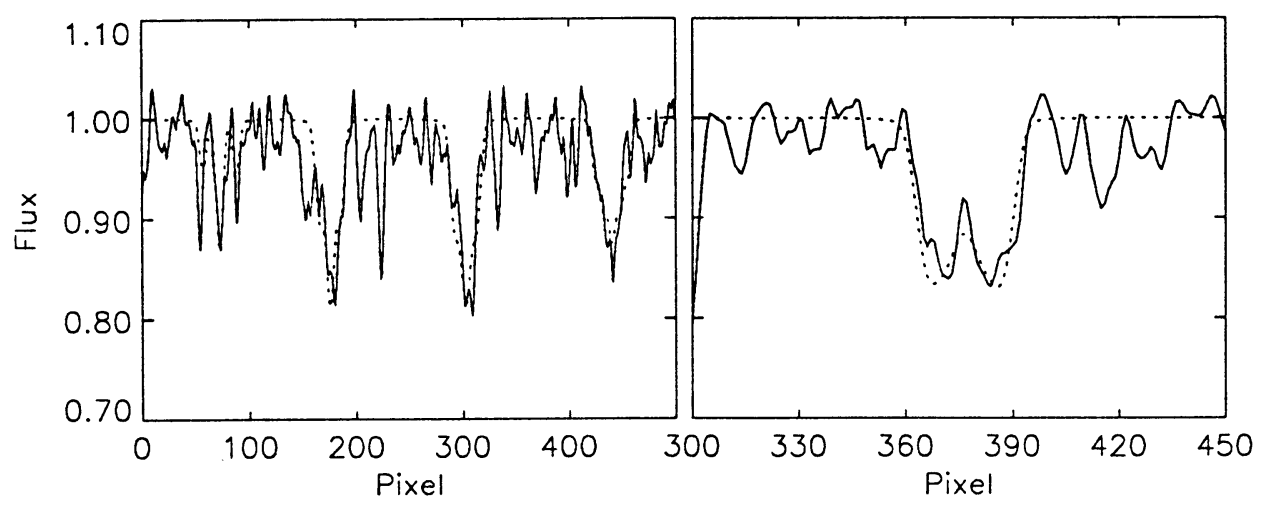

Fig. 1. Unapodized, smoothed line profiles from AU Mic (left; 4,480, 4,488, 4,496, 4,501 $\mathrm{cm}^{-1} ; g_{\text {eff }}=2.5,1.58,1.67$, and 2.0 , respectively; $0.16 \AA /$ pixel) and EV Lac (right; $4,496 \mathrm{~cm}^{-1}$ only). The lines have been "spliced together" for convenient display. The fits (dashed) are for $B=4.2 \mathrm{kG}$ with $f=0.55$ (AU Mic) and $B=4.3 \mathrm{kG}$ with $f=0.85$ (EV Lac).

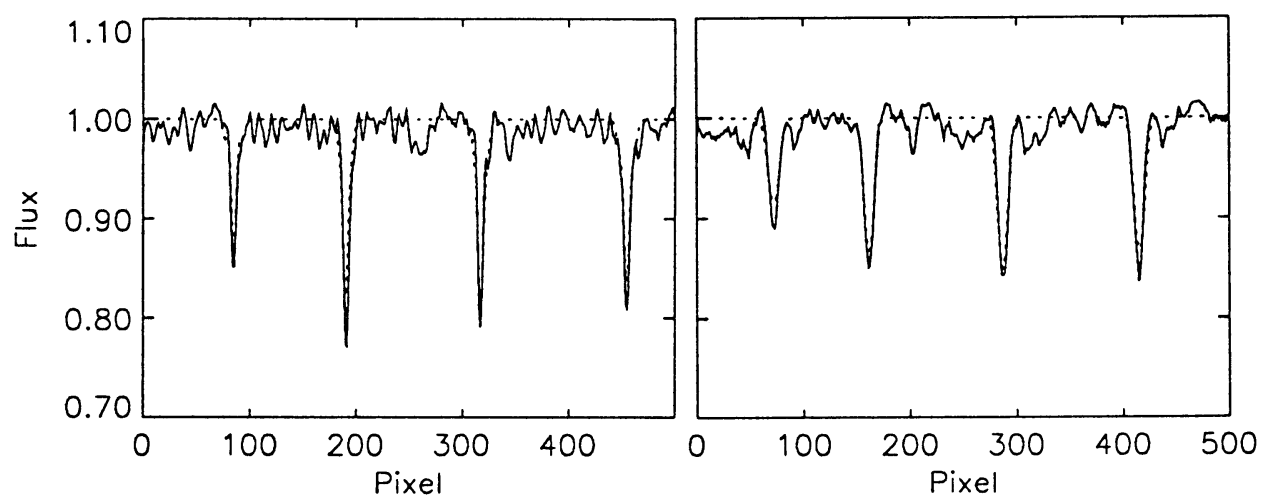

Fig. 2. Similar to Figure 1, but for $\xi$ Boo B (left: fit with $B=2.3 \mathrm{kG}$ and $f=0.20$ ) and HR 7275 (right: fit with $B=1.4 \mathrm{kG}$ and $f=0.40$ ). 
shallow to have significant opacity broadening, leaving magnetic fields as the only plausible explanation. In more rapidly rotating RS CVns, the effect of magnetic fields is subtle, appearing as slightly enhanced equivalent widths in lines with many and/or well-separated Zeeman components (e.g., Basri, Marcy, and Valenti 1992).

The models were calculated with a full-disk integration that partitions $\mu$ (the cosine of the emergence angle) into 5 intervals, and includes radial-tangential macroturbulence. Local line profiles were computed using the Unno-Rachkovsky radiative transfer solution (Unno 1956), including magneto-optical effects (see Saar et al. 1990). Line opacities were constrained to have their predicted (Kurucz and Peytremann 1975) ratios, and areas affected by blends (as determined from the comparison spectra) were given less weight. I adopted previous $v \sin i$ values where available. For ease of comparison with previous results, we used a simple two component model: The observed flux, $F$, is modeled as

$$
F=f F_{\text {mag }}(B)+(1-f) F_{\text {nonmag }}(B=0),
$$

where $B$ is the average field strength of active regions and $f$ is their fractional area coverage (filling factor). Fields were assumed to be homogeneously distributed and $F_{\text {mag }}(0)=F_{\text {nonmag }}(0)$.

No horizontal $B$ distributions or vertical gradients were considered, although there is some evidence for both in dMe stars (Saar 1992a). The effect of a significant vertical gradient in $B$ was tested for AD Leo, using a simple canopy, with $B$ increasing exponentially with depth (Saar 1992a). In this model, the FWHM of the $B$ distribution is $\sim 4 \mathrm{kG}$, about the same as $B$ is in the model in which it is constant with height, although the average, $\bar{B}$, in this model is lower, $\sim 3.2 \mathrm{kG}$. The filling factor, $f$, on the other hand, is reduced by nearly a factor of two, from $65 \%$, to $38 \%$.

AU Mic, AD Leo, and EV Lac have had previous magnetic measurements and $\xi$ Boo B had an upper limit (Saar and Linsky 1985; Saar, Linsky, and Giampapa 1987; Saar 1990). Using the new, better quality data now available and the more realistic line models described above, I have derived improved $f$ and $B$ values for these stars (Table 1 and Figs. 1 and 2). In general, $f$ values are somewhat reduced from previous measurements. An upper limit can be set of $f$ for HD 156026, a moderately active $\mathrm{K}$ dwarf rotating two times slower than $\xi$ Boo B. Marginal detections of magnetic flux have been made in two RS CVn stars: $\sigma$ Gem and HR 7275 (Fig. 2). No clear evidence for strong fields $(B \geq 4 \mathrm{kG})$ are seen on these stars. Assuming a ratio $I_{C \text {,spot }} / I_{C \text {,quiet }}=0.7$ (Saar and Linsky 1985), we can thus rule out high- $B$ umbrae with $f_{\text {spot }} \gtrsim 0.20$ on either star. The equivalent width anomalies due to the magnetic intensification effect (see Leroy 1962; Basri et al. 1992) and slight line shape changes can be modeled well with fairly widespread fields in the 1-1.5 kG range. Due to their rapid rotation, $f$ and $B$ are poorly separated for the RS CVn stars; the product $f B$ is the best determined quantity (see also Saar, Piskunov, and Tuominen 1992).

The stronger $B$ seen in the $\mathrm{M}$ dwarfs relative to $\xi$ Boo $\mathrm{B}$ is consistent with previous results (e.g., Saar and Linsky 1985; Saar et al. 1987). Taken together with the low $B$ measured for the RS CVn stars, this result adds further evidence for a limit on $B$ in the non-spot stellar active regions which is dependent on both $\mathrm{T}_{\mathrm{eff}}$ and 
TABLE I

New IR Measurements of Stellar Magnetic Parameters

\begin{tabular}{lcccccccc}
\hline Star & Sp. Type & $\begin{array}{c}\mathrm{R}-\mathrm{I} \\
(\mathrm{B}-\mathrm{V})\end{array}$ & $\mathrm{S} / \mathrm{N}^{a}$ & $\begin{array}{c}B \\
(\mathrm{kG})\end{array}$ & $\begin{array}{c}f \\
(\%)\end{array}$ & $\begin{array}{c}\text { adopted } \\
B_{\text {eq }}(\mathrm{kG})\end{array}$ & $\begin{array}{c}\mathrm{P}_{\text {rot }} \\
(\text { days })\end{array}$ & $\begin{array}{c}\text { est. } \tau_{c} \\
(\text { days })\end{array}$ \\
\hline AU Mic & M1.6Ve & 0.84 & 40 & 4.2 & 55 & 3.4 & 4.65 & 20 \\
AD Leo & M3.5Ve & 1.12 & 100 & 4.0 & 65 & 3.8 & 2.7 & 20 \\
AD Leo & & & & 3.2 & 38 & & & \\
EV Lac & M4.5Ve & 1.15 & 40 & 4.3 & 85 & 4.4 & 4.8 & 20 \\
$\xi$ Boo B & K4V & $(1.17)$ & 90 & 2.3 & 20 & 2.5 & 11 & 20 \\
HD 156026 & K5V & $(1.16)$ & 120 & $\ldots$ & $<20$ & 2.5 & 18.5 & 20 \\
\hline$\sigma$ Gem & K1IIIe & $(1.12)$ & 160 & $1.1:^{c}$ & $70:^{c}$ & 0.7 & 19.4 & $\approx 80$ \\
HR 7275 & K1IV & $(1.09)$ & 190 & $1.4:^{d}$ & $40:^{d}$ & 1.2 & $\approx 28$ & $\approx 50$ \\
\hline
\end{tabular}

${ }^{a}$ per resolution element

${ }^{b} \nabla B$ model with $B_{\min }=2 \mathrm{kG}, \bar{B}=3.2 \mathrm{kG}, B_{\mathrm{fwhm}}=4.3 \mathrm{kG}, \sigma_{B}=2 \mathrm{kG}$.

c uncertain; $f B \approx 0.8 \mathrm{kG}$ is the best determined quantity

${ }^{d}$ uncertain; $f B \approx 0.55 \mathrm{kG}$ is the best determined quantity

luminosity class. The best explanation probably remains the following: $B$ is limited by pressure balance with the ambient gas such that $B \leq B_{\text {eq }}$, where $B_{\text {eq }} \propto P_{\text {gas }}^{0.5}$ is the pressure equipartition field, (e.g., Galloway and Weiss 1981). I have estimated $B_{\text {eq }}\left(\right.$ at $\left.\tau_{5000}=1\right)$ and find that $B$ is generally near or below this value (see Table 1). The total magnetic flux $(\propto f B)$ and $f$, on the other hand, both increase with angular velocity and inverse Rossby number, $\tau_{c} / \mathrm{P}_{\text {rot }}$ (where $\tau_{c}$ is the convective turnover time, taken from Stepien 1989 and Basri 1987). This is again consistent with previous results (e.g., Saar 1990; 1991). The importance of the present results, however, is that they extend the relation to RS CVn's, more rapid rotators, and cool dMe stars. I refer the reader to Saar (1993) for a more complete analysis of these relationships.

\section{Acknowledgements}

This work is based on data obtained at NOAO. I thank K. Hinkle, W. Lenz, P. Russell, and D. Neff for help with the observations, and G. Basri, M. Giampapa, J. Linsky, G. Marcy, and S. Solanki for enlightening discussions. This research is supported by NASA grant NAGW-112, Interagency Transfer W-15130, and NSF grant INT-8900202.

\section{References}

Basri, G.S., Marcy, G.W., Valenti, J.: 1992, Astrophys. J. 390, 622.

Basri, G.S., Marcy, G.W., Valenti, J.: 1990, Astrophys. J. 360, 650.

Basri, G.S.: 1987, Astrophys. J. 316, 377. 
Bopp, B.W., Saar, S.H., Ambruster, C., Feldman, P., Dempsey, R., Allen, M., Barden, S.P.: 1989, A strophys. J. 339, 1059.

Galloway, D.J., Weiss, N.O. 1981, Astrophys. J. 243, 945.

Giampapa, M.S. 1985, Astrophys. J. 299, 781.

Giampapa, M.S., Golub, L., Worden, S.P.: 1983, Astrophys. J. (Letters) 268, 121.

Hall, D.N.B., Ridgway, S., Bell, E.A., Yarborough, J.M. 1979, S.P.I.E., 172, 121.

Kurucz, R., Peytremann, E.: 1975, A Table of Semiempirical gf Values, SAO Rep. 362.

Leroy, J.L.: 1962, Ann. d'Ap., 25, 127.

Marcy, G.W., Bruning, D.H.: 1984, Astrophys. J. 281, 286.

Saar, S.H.: $1992 a$, in Cool Stars, Stellar Systems, and the Sun, M. Giampapa and J. Bookbinder (eds.), ASP Conf. Ser. 26, p. 252.

Saar, S.H.: 1993, these proceedings.

Saar, S.H.: 1991, in The Sun and Cool Stars: Activity, Magnetism, Dynamos, I. Tuominen et al. (eds.), Springer, Berlin, p. 389.

Saar, S.H.: 1990, in The Solar Photosphere: Structure, Convection, and Magnetic Fields, J. O. Stenflo (ed.), Kluwer, Dordrecht, p. 427.

Saar, S.H., Golub, L., Bopp, B.W., Herbst, W., Huovelin, J.: 1990, in Evolution in Astrophysics, E. Rolfe (ed.), ESA SP-130, p. 431.

Saar, S.H., Linsky, J.L., Giampapa, M.S.: 1987, in 27th Liège Astrophys. Colloq., L. Delbouille and A. Monfils (eds.), U. de Liège, Liège, p. 103.

Saar, S.H., Linsky, J.L.: 1985, A strophys. J. (Letters) 299, L47.

Saar, S.H., Piskunov, N.E., Tuominen, I.: 1992, in Cool Stars, Stellar Systems, and the Sun, M. Giampapa and J. Bookbinder (eds.), ASP Conf. Ser. 26, p. 255.

Stepień, K.: 1989, Astron. Astrophys. 210, 273.

Strassmeier, K.G., et al. : 1988, Astron. Astrophys. Suppl. 72, 291.

Unno, W.: 1956, Pub. Astron. Soc. Japan 8, 108.

Vogt, S.S., Soderblom, D.R., Penrod, G.D.: 1983, Astrophys. J. 269, 250. 